International Journal of Algebra, Vol. 7, 2013, no. 8, 349 - 354

HIKARI Ltd, www.m-hikari.com

\title{
Gamma Invariants and the Torsion-Freeness of Ext
}

\author{
Ulrich Albrecht \\ Department of Mathematics, Auburn University \\ Auburn, AL 36849, USA \\ albreuf@auburn.edu \\ Stefan Friedenberg \\ Fachbereich Mathematik \\ Universität Duisburg-Essen \\ 45320 Essen, Germany \\ stefan.friedenberg@uni-duisburg-essen.de
}

Copyright (c) 2013 Ulrich Albrecht and Stefan Friedenberg. This is an open access article distributed under the Creative Commons Attribution License, which permits unrestricted use, distribution, and reproduction in any medium, provided the original work is properly cited.

Abstract. This paper investigates homological properties of the class ${ }^{*} B$ of Abelian groups $A$ such that $\operatorname{Ext}(A, B)$ is torsion-free. In particular, our results demonstrate that Gamma-invariants cannot be introduced in a meaningful way for ${ }^{*} B$.

\section{Mathematics Subject Classification: 20K15}

Keywords: A-Solvable Group, Flat, Endomorphism Ring

Ascending chains of subgroups have always been an the essential tool in the study of infinite Abelian groups. This was further emphasized by Shelah's solution of the Whitehead problem and a series of related papers concerning the invariants of the divisible group $\operatorname{Ext}(A, B)$ in the case that $A$ and $B$ are torsionfree Abelian groups. In particular, either $\operatorname{Ext}(A, B)=0$ or $r_{0}(\operatorname{Ext}(A, B))=$ $2^{\aleph_{0}}$ if $A$ and $B$ are countable. On the other hand, the Ext-group may be torsion-free without vanishing. 
To simplify our notation, we denote the class of all torsion-free Abelian groups $A$ such that $\operatorname{Ext}(A, B)=0$ by ${ }^{\perp} B$, while ${ }^{*} B$ consists of the groups $A$ for which $\operatorname{Ext}(A, B)$ is torsion-free. Clearly, ${ }^{\perp} B \subseteq{ }^{*} B$. Moreover, since ${ }^{\perp} B$ was successfully investigated using Eklof's $\Gamma$-invariants [7], it can be expected that these invariants play a similar role in the apparently related discussion of ${ }^{*} B$. One of the consequences of our discussion is the surprising fact that this is not the case.

To define the $\Gamma$-invariant of an Abelian group $A$ of regular cardinality $\kappa$ with respect to a property $\mathcal{P}$, write $A=\cup_{\alpha<\kappa} A_{\alpha}$ where the $A_{\alpha}$ 's form a smooth ascending chain of subgroups of $A$ with $A_{0}=\{0\}$ and $\left|A_{\alpha}\right|<\kappa$ for all $\alpha$, and consider $S=\left\{\alpha<\kappa \mid A_{\beta} / A_{\alpha}\right.$ does not satisfy $\mathcal{P}$ for some $\left.\beta>\alpha\right\}$. The Gamma-invariant $\Gamma_{\kappa, P}(A)$ of $A$ with respect to $\mathcal{P}$ is the equivalence class of $S$ with respect to the intersection with closed and unbounded sets.

Surprisingly, we encounter immediate difficulties when attempting to use these invariants for the property $A \in{ }^{*} B$. Looking at ${ }^{\perp} B$, we realize that its closure with respect to extensions is what enables the use of $\Gamma$-invariants. In contrast, ${ }^{*} B$ need not be closed with respect to extensions even in the case $B=\mathbb{Z}$ :

Example 1. If $0 \rightarrow \mathbb{Z} \rightarrow A \rightarrow \mathbb{Q} \rightarrow 0$ represents a non-zero element of $\operatorname{Ext}(\mathbb{Q}, \mathbb{Z})$, then $\operatorname{Ext}(A, Z)$ is not torsion-free.

Proof. Clearly, $\operatorname{Hom}(A, \mathbb{Z})=0$. Therefore, the induced sequence

$$
0=\operatorname{Hom}(A, \mathbb{Z}) \rightarrow \operatorname{Hom}(\mathbb{Z}, \mathbb{Z}) \rightarrow \operatorname{Ext}(\mathbb{Q}, \mathbb{Z}) \rightarrow \operatorname{Ext}(A, \mathbb{Z})
$$

is exact. If $\operatorname{Ext}(A, \mathbb{Z})$ were torsion-free, then $\operatorname{Hom}(\mathbb{Z}, \mathbb{Z})$ would be isomorphic to a pure subgroup of the torsion-free divisible group $\operatorname{Ext}(\mathbb{Q}, \mathbb{Z})$, which is impossible.

However, ${ }^{*} B$ is closed with respect to $B$-cobalanced extensions, where a sequence $0 \rightarrow U \rightarrow G \rightarrow H \rightarrow 0$ is $B$-cobalanced if $B$ is injective with respect to it, i.e. the induced sequence $0 \rightarrow H^{*} \rightarrow G^{*} \rightarrow U^{*} \rightarrow 0$ is exact. Here, the symbol ( $)^{*}$ denotes one of the contra-variant functors $G^{*}=\operatorname{Hom}(G, B)$ and $M^{*}=\operatorname{Hom}_{E}(M, B)$ between the category of Abelian groups and the category of left $E$-modules. Moreover, $\psi_{G}$ is the induced canonical map $G \rightarrow G^{* *}$. For details, see [12]. For instance, every sequence $0 \rightarrow U \rightarrow G \rightarrow H \rightarrow 0$ with $G \in{ }^{\perp} B$ is $B$-cobalanced. Unfortunately, there is no direct connection between the groups in a short exact sequence belonging to ${ }^{*} B$ and the sequence being $B$-cobalanced as the following example shows:

Example 2. a) There exists group $G_{1} \subseteq G_{2}$ such that $G_{1}, G_{2}$ and $G_{2} / G_{1}$ belong to ${ }^{*} \mathbb{Z}$, but the sequence $0 \rightarrow G_{1} \rightarrow G_{2} \rightarrow G_{2} / G_{1} \rightarrow 0$ is not $\mathbb{Z}$-cobalanced.

b) There exists a group $G \in{ }^{*} \mathbb{Z}$ such that 0 and $G$ are the only $\mathbb{Z}$-cobalanced subgroups of $G$. 
Proof. For a), let $G$ be an Abelian group such that $\operatorname{Ext}(G, \mathbb{Z})$ is non-zero and torsion-free. A free resolution $0 \rightarrow F_{1} \rightarrow F_{2} \rightarrow G \rightarrow 0$ of $G$ induces the sequence $F_{2}^{*} \rightarrow F_{1}^{*} \rightarrow \operatorname{Ext}(Z, \mathbb{Z}) \rightarrow 0$. Since $\operatorname{Ext}(G, \mathbb{Z}) \neq 0$, the original sequence cannot be $\mathbb{Z}$-cobalanced. To see b), consider $G=\mathbb{Q}$.

We nevertheless obtain the following

Lemma 3. Let $0 \rightarrow U \stackrel{\alpha}{\rightarrow} G \stackrel{\beta}{\rightarrow} H \rightarrow 0$ be an exact sequence of torsion-free Abelian groups.

a) If $G \in{ }^{*} B$, then so is $U$.

b) If the sequence is a $B$-cobalanced, then $G \in{ }^{*} B$ if and only if $U, H \in{ }^{*} B$.

Proof. Consider the induced sequence

$$
\operatorname{Ext}(H, B) \stackrel{\beta^{*}}{\rightarrow} \operatorname{Ext}(G, B) \rightarrow \operatorname{Ext}(U, B) \rightarrow 0 .
$$

Since $H$ is torsion-free, $i m\left(\beta^{*}\right)$ is divisible. Hence a) holds. Moreover, $\beta^{*}$ is one-to-one if the original sequence is $B$-cobalanced, from which b) follows directly.

We say that a torsion-free Abelian group $A$ of (regular) cardinality $\kappa$ has a $B$-cobalanced $\kappa$-filtration if $A=\cup_{\alpha<\kappa} A_{\alpha}$ such that $\left|A_{\alpha}\right|<\kappa$ and $A_{\alpha}$ is $B$ cobalanced in $A_{\alpha+1}$ for all $\alpha<\kappa$. Furthermore, the group $A$ is $B$-generated if there exists an exact sequence $\oplus_{I} B \rightarrow A \rightarrow 0$. If $A$ has regular cardinality $\kappa$, and $|B|<\kappa$, then $A$ is $B$-generated if and only if has a $\kappa$-filtration of $B$-generated subgroups.

Before continuing, we want to remind the reader of some terminology. For every Abelian group $B$ with endomorphism ring $E(B)$, we have a pair $\left(H_{B}, T_{B}\right)$ of adjoint functors between the category of Abelian groups and the category of right $E(B)$-modules defined by $H_{B}(G)=\operatorname{Hom}(B, G)$ and $T_{B}(M)=M \otimes_{E(B)} B$ for all Abelian groups $G$ and all right $E(B)$-modules $M$. Associated with these functors are natural maps $\theta_{G}: T_{B} H_{B}(G) \rightarrow G$ and $\phi_{M}: M \rightarrow H_{B} T_{B}(M)$. The category of $B$-solvable groups consists of all groups $G$ for which $\theta_{G}$ is an isomorphism. Moreover, $G$ is $B$-projective if it isomorphic to a direct summand of $\oplus_{I} B$ for some index-set $I$. If $B$ is a torsion-free Abelian group of finite rank, then $H_{B}$ and $T_{B}$ induce an equivalence between the category of $B$-projective groups and the category of projective right $E(B)$-modules. Finally, a torsionfree group of finite rank is a finitely faithful $S$-group if $r_{p}(E)=\left[r_{p}(A)\right]^{2}$ where $r_{p}(G)=\operatorname{dim}_{\mathbb{Z} / p \mathbb{Z}} G / p G$ denotes the $p$-rank of a torsion-free group $G$. Arnold showed in [6] that a torsion-free group $B$ of finite rank is a finitely faithful $S$-group if an only if $B \in{ }^{*} B$. Furthermore, every finitely faithful $S$-group has a hereditary endomorphism ring.

Theorem 4. Let $B$ be a torsion-free group.

a) If $A$ is a torsion-free group which has a $\kappa$-filtration $\left\{A_{\alpha}\right\}_{\alpha<\kappa}$ of subgroups $A_{\alpha}$ such that $S=\left\{\alpha<\kappa \mid A_{\alpha+1} / A_{\alpha} \notin{ }^{*} B\right\}$ is not stationary, then $A \in{ }^{*} B$. 
b) Let B a finitely faithful S-group. A reduced B-generated group A of cardinality $\aleph_{1}$ has a $B$-cobalanced filtration of countable subgroups with factors in ${ }^{*} B$ if and only if $A$ is $B$-projective.

Proof. a) Since $S$ is not stationary, there is a closed and unbounded subset $C$ of $\kappa$ with $S \cap C=\emptyset$. Considering the $\kappa$-filtration $\left\{A_{\alpha}: \alpha \in C\right\}$ of $A$ permits to assume that $A_{\alpha+1} / A_{\alpha} \in{ }^{*} B$ for all $\alpha$. Observe that multiplication by a prime $p$ induces the exact sequence

$$
\operatorname{Hom}(A, B) \rightarrow \operatorname{Hom}(A, B / p B) \rightarrow \operatorname{Ext}(A, B) \rightarrow \operatorname{Ext}(A, B)
$$

where the last map is multiplication by $p$. Therefore, $\operatorname{Ext}(A, B)$ is torsion-free, once we have shown that the first map in this sequence is onto. To see this, let $f \in \operatorname{Hom}(A, B / p B)$. Suppose that $f \mid A_{\alpha} \in \operatorname{Hom}\left(A_{\alpha}, B / p B\right)$ lifts to a map $f_{\alpha} \in \operatorname{Hom}\left(A_{\alpha}, B\right)$. Since $B$ is injective with respect to $0 \rightarrow A_{\alpha} \rightarrow A_{\alpha+1} \rightarrow$ $A_{\alpha+1} / A_{\alpha} \rightarrow 0$, there is a map $g \in \operatorname{Hom}\left(A_{\alpha+1}, B\right)$ with $g \mid A_{\alpha}=\phi_{\alpha}$. We consider the diagram

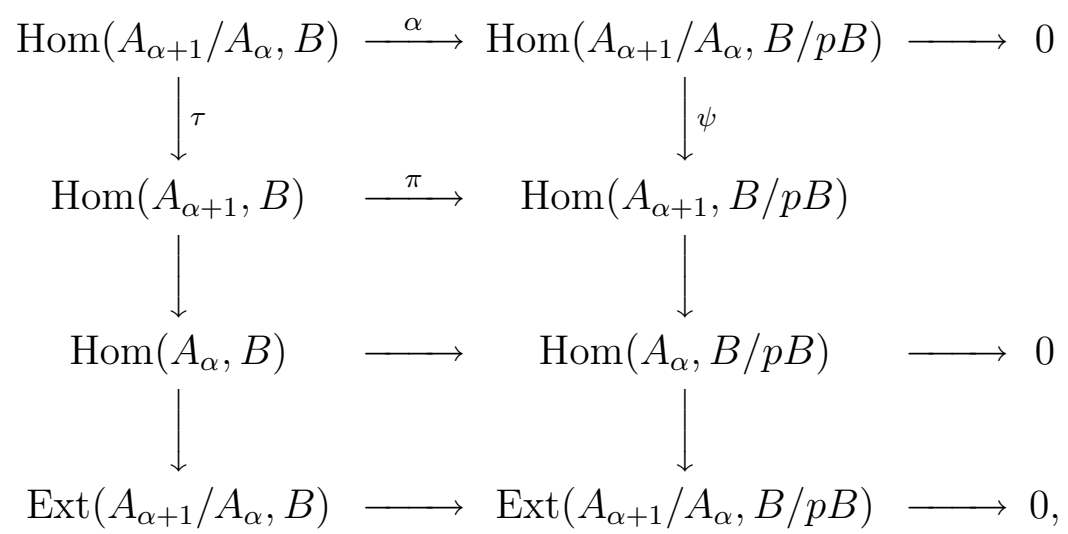

and define a map $\delta: A_{\alpha+1} \rightarrow B / p B$ by $\delta=f \mid A_{\alpha+1}-\pi g$. Because $\delta \mid A_{\alpha}=0$, we obtain $\delta \in \operatorname{Im}(\psi)$. Since $\alpha$ is onto, there exists a map $h \in \operatorname{Hom}\left(A_{\alpha+1} / A_{\alpha}, B\right)$ with $\psi(\alpha(h))=\delta$. Define $\lambda: A_{\alpha+1} \rightarrow B$ by $\lambda=\tau(h)$, and obtain $\pi \lambda=\delta$ and $\lambda \mid A_{\alpha}=0$. Now set $f_{\alpha+1}=g+\lambda$ because of $f_{\alpha+1} \mid A_{\alpha}=f_{\alpha}$ and $\pi f_{\alpha+1}=$ $\pi g+\delta=f \mid A_{\alpha+1}$. We now define $\bar{f}$ by $\bar{f}(a)=f_{\alpha}(a)$ if $a \in A_{\alpha}$, and obtain $\pi \bar{f}=f$.

b) Suppose that $A$ has a $B$-cobalanced filtration $\left\{A_{\alpha} \mid \alpha<\omega_{1}\right\}$ of countable subgroups. Observe that each $A_{\alpha} \in{ }^{*} B$ by Lemma 3 if $\alpha=\beta+1$, or of part a) of Theorem 4 if $\alpha$ is a limit ordinal. For each countable subset $Y$, there is a countable subset $X$ of $\operatorname{Hom}(B, A)$ such that $Y \subseteq X B$ since $A$ is $B$-generated. Because $B$ is countable, $A$ is the union of a smooth chain $\left\{C_{\alpha} \mid \alpha<\omega_{1}\right\}$ of countable, $B$-generated subgroups. Since there exists a closed and unbounded subset $E$ of $\omega_{1}$ such that $C_{\alpha}=A_{\alpha}$ for all $\alpha \in E$, we may assume that each $A_{\alpha}$ is $A$-generated.

Since $A_{\alpha}$ is $B$-generated, it is a $B_{0}$-module where $B_{0}$ is the subring of $\mathbb{Q}$ generated by $\left\{\frac{1}{p} \mid B=p B\right\}$. Because $A$ is reduced, $A_{\alpha}$ is isomorphic to a subgroups of $B^{I_{\alpha}}$ for some index-set $I_{\alpha}$ by [3, Proposition 2.2]. Since $E(B)$ is 
right hereditary, it is also right and left Noetherian by [12], and $H_{B}\left(B^{I_{\alpha}}\right)$ is $\aleph_{1}$-projective. By [2], $A_{\alpha}$ is $B$-solvable, and $H_{B}\left(A_{\alpha}\right)$ is a countable submodule of $H_{B}\left(B^{I_{\alpha}}\right)$. Hence, $A_{\alpha} \cong T_{B} H_{B}\left(A_{\alpha}\right)$ is $B$-projective.

The sequence $0 \rightarrow\left(A_{\alpha+1} / A_{\alpha}\right)^{*} \rightarrow A_{\alpha+1}^{*} \rightarrow A_{\alpha}^{*} \rightarrow 0$ is exact and induces the top-row of the diagram

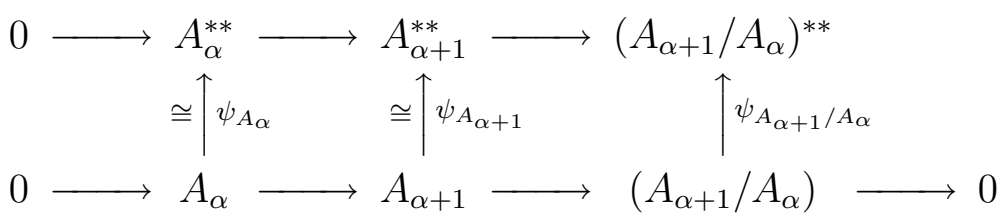

where the first two maps are isomorphisms since $B$ is slender. By the Snake Lemma, $\psi_{A_{\alpha+1} / A_{\alpha}}$ is a monomorphism. Thus, $A_{\alpha+1} / A_{\alpha}$ can be embedded into a group of the form $B^{I}$. Arguing as before, we obtain that $A_{\alpha+1} / A_{\alpha}$ is $B$ projective. Since $E(B)$ is hereditary, the every submodule of the last module in the induced sequence $0 \rightarrow H_{B}\left(A_{\alpha}\right) \rightarrow H_{B}\left(A_{\alpha+1}\right) \rightarrow H_{B}\left(A_{\alpha+1} / A_{\alpha}\right)$ is projective, and the top row of the diagram

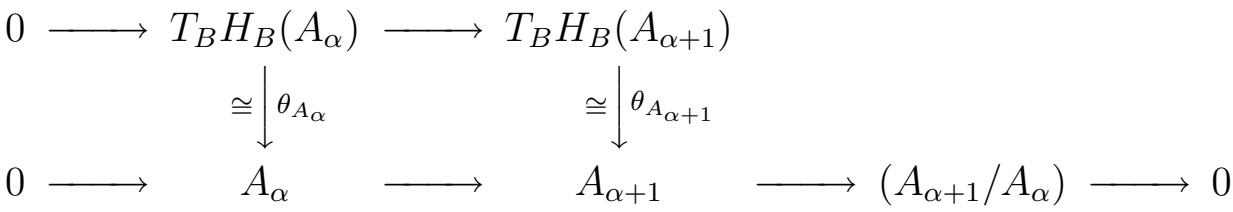

splits. Thus, the bottom row splits too, say $A_{\alpha+1}=A_{\alpha} \oplus U_{\alpha}$. Therefore, $A \cong \oplus_{\alpha} U_{\alpha}$ is $B$-projective.

Conversely, observe that a $B$-projective group $A$ is of the form $A=\oplus_{\alpha<\omega_{1}} B_{\alpha}$ where each $B_{\alpha}$ is isomorphic to a $B$-generated subgroup of $B$ since $E$ is right hereditary. Clearly, $A_{\alpha}=\oplus_{\beta<\alpha} B_{\beta}$ is a $B$-cobalanced filtration.

Corollary 5. Let $B$ be a finitely faithful $S$-group. If $A$ is an $\aleph_{1}$-B-projective group of cardinality $\aleph_{1}$, which is not B-projective, then $A$ does not have a $B$-cobalanced $\aleph_{1}$-filtration with factors in ${ }^{*} B$.

By the well-known Corner-Dugas-Goebel construction, there exists an $\aleph_{1}$ free left $E^{o p}$-module $M$ such that $E n d_{\mathbb{Z}}\left(M^{+}\right)$is $E^{o p}$. Viewing $M$ as a right $E$-module gives an $\aleph_{1}$-projective $E$-module which is not projective. By [1], $\phi_{M}$ is an isomorphism, and $A=T_{B}(M)$ is an $\aleph_{1}$ - $B$-projective, $B$-solvable Abelian group which does not have a $B$-balanced $\aleph_{1}$-filtration.

Furthermore, we obtain

Corollary 6. The following statements are undecidable in ZFC:

a) If $G$ has cardinality $\aleph_{1}$ and $\operatorname{Ext}(G, \mathbb{Z})$ is torsion-free, then $G$ has a $\mathbb{Z}$ cobalanced filtration of countable subgroups;

b) There exists a reduced group $H$ with $\operatorname{Ext}(H, \mathbb{Z})$ torsion-free and for which one can find an exact sequence $0 \rightarrow \mathbb{Z} \rightarrow G \rightarrow H \rightarrow 0$ with $\operatorname{Ext}(G, \mathbb{Z})$ is not torsion-free. 
Proof. Assume $V=L$. By [7, Cor. XII, 2.11] there exists a coseparable group $G$ of cardinality $\aleph_{1}$, which is not free. Since Whitehead-groups are free, $\operatorname{Ext}(G, \mathbb{Z}) \neq 0$. By Theorem $4 G$ does not have a $\mathbb{Z}$-balanced filtration and if $0 \rightarrow \mathbb{Z} \rightarrow X \rightarrow G \rightarrow 0$ represents a non-zero element of $\operatorname{Ext}(G, \mathbb{Z})$, then $\operatorname{Ext}(X, \mathbb{Z})$ is not torsion-free arguing as in the proof of Example 1.

On the other hand we assume that the existence of supercompact cardinals is consistent with ZFC. Then it is also consistent with ZFC that every coseparable group is free, cf. [13]. Thus $\operatorname{Ext}(H, \mathbb{Z})$ torsion-free implies that $H$ is free. Clearly, free groups have $\mathbb{Z}$-cobalanced filtrations and if $\operatorname{Ext}(H, \mathbb{Z})$ is torsionfree, then $0 \rightarrow \mathbb{Z} \rightarrow G \rightarrow H \rightarrow 0$ splits.

\section{REFERENCES}

[1] U. Albrecht; Endomorphism rings and A-projective torsion-free Abelian groups; Abelian Group Theory, Proceedings Honolulu 1982/83; Springer Lecture Notes in Mathematics 1006; Springer Verlag; Berlin, New York, Heidelberg (1983); 209-227.

[2] U. Albrecht; Endomorphism Rings of Faithfully Flat Abelian Groups; Resultate der Mathematik 17 (1990); 179 - 201.

[3] Albrecht, U., and Friedenberg, S.; Murley groups and the torsion-freeness of Ext; preprint.

[4] Albrecht, U., and Goeters, H. P.; Strong S-groups; Colloquium Mathematicum 80 (1999); 97-105.

[5] Arnold, D. M.; Finite Rank Torsion Free Abelian Groups and Rings, Lecture Notes in Mathematics 931; Springer-Verlag Berlin-Heidelberg-New York (1982).

[6] Arnold, D. M.; Endomorphism rings and subgroups of finite rank torsion-free Abelian groups; Rocky Mountain J. of Math. 12(2) (1982); 241-256.

[7] Eklof, P. C., Mekler, A. H.; Almost Free Modules; Vol. 46; North Holland Mathematical Library (1990).

[8] Faticoni,T. G., and Goeters, H. P.; On torsion-free Ext; Comm. in Alg. 16(9) (1988); 1853-1876.

[9] Fuchs, L.; Infinite Abelian Groups Vol. 1/2; Academic Press; New York and London (1970/73).

[10] Goeters, H. P.; When is $\operatorname{Ext}(A, B)$ torsion-free? and related problems; Comm. in Alg. 16(8) (1988); 1605-1619.

[11] Goeters, H. P.; Extensions of finitely faithful $S$-groups; Lecture Notes in Pure and Applied Mathematics 182; Marcel Dekker; New York (1996); 273 - 284.

[12] Huber, M., and Warfield, R. B.; Oberwolfach 876 (1981).

[13] Mekler, A. H., and Shelah, S.; Every coseparable group may be free; Israel J. of Math. 81 (1993); 161-178.

[14] Stenström, B.; Rings of Quotients; Lecture Notes in Math. 217; Springer Verlag, Berlin, Heidelberg, New York (1975).

\section{Received: February 27, 2013}

